\title{
Avaliação econômica sob condições de risco em sistema produtivo de gado de leite na região Norte do estado do Rio de Janeiro
}

[Economic viability under risk condictions of a milk production system in North Region of Rio de Janeiro State]

\author{
I.R. Haddade ${ }^{1,3}$, P.M. Souza ${ }^{2}$, E.E.L. Barros ${ }^{1}$, G.R. Alves ${ }^{1}$, L. Scolforo ${ }^{1}$, \\ M.D. Cordeiro ${ }^{1}$, A.A.C. Peres ${ }^{1}$, L.T. Henriques ${ }^{1}$ \\ ${ }^{1}$ Doutorando em Produção Animal - Laboratório de Zootecnia e Nutrição Animal \\ Centro de Ciências e Tecnologias Agropecuárias \\ Av. Alberto Lamego, 2000 - Parque Califórnia \\ 28015-620 - Campos dos Goytacazes, RJ \\ ${ }^{2}$ Laboratório de Engenharia Agricola - CCTA - UENF \\ ${ }^{3}$ Bolsista da CAPES
}

\begin{abstract}
RESUMO
Avaliou-se financeiramente um modelo de sistema produtivo de leite produzido em pasto na região Norte Fluminense - RJ. Foi elaborada uma planilha de fluxo de caixa (horizonte de 20 anos), a partir da qual calcularam-se os indicadores: valor presente líquido (VPL a 6 e 10\%) e taxa interna de retorno (TIR). Cada fator envolvido nas receitas ou nas despesas do projeto foi submetido à análise de sensibilidade para que fossem identificados aqueles com maiores pesos na redução dos indicadores calculados. Realizou-se simulação de Monte Carlo, visando avaliar a probabilidade de ocorrência para determinados valores financeiros associados ao risco de divergências nos itens do projeto. Os resultados demonstraram viabilidade financeira para o sistema proposto (VPL a 6 e 10\%, R $\$ 197.880,00$ e $\mathrm{R} \$ 8.067,00$, respectivamente, e TIR 10,2\%). A rentabilidade foi afetada, em ordem decrescente, pelos fatores: preço do leite, aquisição de vacas nos primeiros anos do projeto, preço das vacas descartadas, mão-de-obra e preço do concentrado para os animais adultos. Os resultados da simulação de Monte Carlo demonstraram elevada probabilidade $(39 \%)$ de a rentabilidade do projeto ser inferior ao rendimento da poupança, representando alto risco na adoção do sistema.
\end{abstract}

Palavras-chave: produção de leite, sistema em pasto, análise de sensibilidade, simulação de Monte Carlo, taxa interna de retorno, valor presente líquido

\begin{abstract}
The study aimed to evaluate milk production under grazing system in the North Region of Rio de Janeiro State. The net present worth (NPWs of 6 and 10\%) and internal rate of return (IRR) were calculated from the cash flow chart on a 20-year basis. Sensitivity analyses were performed to evaluate the effect of the income or expense factors on the net present worth (6 and 10\%) and the internal rate of return. A Monte Carlo Method was carried out to evaluate the probabilities of occurrence for certain financial values associated to the risk of deviation in the project itens. The results showed financial feasibility for the proposed system (NPWs of $6 \%$ and 10\% $R \$ 197,880.00$ and $R \$ 8,067.00$, respectively; IRR 10.2\%). The profitability was affected in decreasing order by the following factors: price of milk, purchase of cows in the first years of the project, price of culled cows, labor and the price of the concentrate for adult animals. The results of the Monte Carlo simulation indicated high probabilities (39\%) of the profitability
\end{abstract}

Recebido para publicação em 22 de dezembro de 2003

Recebido para publicação, após modificações, em 17 de julho de 2004

E-mail: haddade@uenf.br 
to be smaller than the interest of the saving account, showing high economic risk of the production system.

Keywords: milk production, grazing system, sensitivity analysis, Monte Carlo method, internal rate of return, net present worth

\section{INTRODUÇÃO}

O planejamento produtivo e o uso de técnicas adequadas de exploração representam a melhor opção para prosperidade em um sistema de produção de leite. Assim, o uso de tecnologia apropriada às diferentes regiões é essencial para o sucesso financeiro da atividade.

Conforme Silva e Passanezi (1998), dependendo do perfil idealizado, a obtenção de bons níveis produtivos e lucratividade aceitável não é tarefa difícil, tornando a atividade competitiva do ponto de vista financeiro. Dessa maneira, merecem destaque estudos que visem não só ao aumento em produtividade, mas também à avaliação financeira com o uso de novas tecnologias.

Segundo Potter et al. (2000), a maioria dos trabalhos científicos não avalia a viabilidade financeira das novas tecnologias estudadas, havendo pouca informação sobre seus benefícios na lucratividade dos sistemas de produção agropecuária.

Neste contexto, destaca-se a importância da análise financeira de projetos agropecuários (Noronha, 1987), que permite avaliar a compensação do investimento em determinada atividade (Schreiner, 1989), gerar informações sobre a relevância financeira dos diversos itens do projeto (Melo et al., 1999) e avaliar o risco de insucesso com a adoção de um determinado sistema mediante o uso da técnica da simulação de Monte Carlo (Azevedo Filho, 1988; Lopes, 1992; Turban, 1993).

Melo et al. (1999) descreveram a análise de sensibilidade como o impacto de variações pessimistas, isoladas em cada item do projeto, sobre sua rentabilidade. De posse da medida desses impactos, são identificados os itens que apresentam maior participação na rentabilidade do sistema produtivo, que, a partir de então, passariam a ser considerados como variáveis aleatórias, permitindo analisar o efeito de diversos cenários alternativos para o sistema. Por meio do método de Monte Carlo, pode-se ainda aferir a probabilidade matemática da ocorrência de determinado valor para o indicador financeiro calculado (Turban, 1993).

Este trabalho teve o objetivo de avaliar a viabilidade econômica e o grau de risco associado de um modelo de sistema de produção de leite em pasto, proposto para a Região Norte Fluminense, em consonância com suas características edafo-climáticas, econômicas e sociais.

\section{MATERIAL E MÉTODOS}

A primeira etapa do trabalho consistiu em um estudo das características da Região Norte Fluminense-RJ, obtendo-se informações para a descrição de um modelo potencial de sistema produtivo. As informações relativas ao sistema foram resumidas na Tab. 1.

Após a definição do perfil a ser estudado, elaborou-se uma planilha de fluxo de caixa, com as quantidades e preços de cada item do sistema, em um horizonte de planejamento para 20 anos. $\mathrm{Na}$ construção da planilha, foi considerada a estabilização de um rebanho de 145 vacas adultas em idade de produção, levando-se em conta índices zootécnicos compatíveis com a realidade do sistema proposto. Na composição dos índices, partiu-se do princípio da não-existência prévia do sistema, sendo adquiridos todos os itens necessários ao seu desenvolvimento. 
Tabela 1. Características gerais da região estudada e alguns índices técnicos utilizados no sistema de produção de leite proposto

\begin{tabular}{lc} 
Características da região & Descrição \\
\hline Tamanho médio da propriedade leiteira & $40-80$ hectares \\
Padrão racial dos animais & $1 / 2 \mathrm{a} 3 / 4 \mathrm{HZ}$ \\
Volumoso (águas) & Pasto \\
Volumoso (seca) & Cana-de-açúcar \\
Irrigação de pastagens & Possível \\
Índices técnicos & \\
Produção diária por vaca em lactação (kg de leite/vaca dia) & 10 \\
Intervalo entre partos (dias) & 390 \\
Período de lactação (dias) & 270 \\
Produção de leite (kg de leite/dia) & 1000 \\
Número médio de vacas adultas no rebanho & 145 \\
\hline
\end{tabular}
$\mathrm{HZ}=$ Holandês $\mathrm{X}$ Zebu.

Para a obtenção dos preços dos itens do projeto, realizou-se uma pesquisa em cinco estabelecimentos comerciais na região, sendo adotados os preços médios praticados. Os valores monetários para os itens de receita, como leite e animais de descarte, foram obtidos a partir de séries históricas extraídas do Ipeadata (Preços..., 2001) e Anualpec (Anuário..., 2001), levando-se em conta os valores relativos à região estudada. Todos esses valores obtidos foram deflacionados utilizando-se o IGP-DI da Fundação Getúlio Vargas, base janeiro de 2002.

Uma vez montado o fluxo de caixa, calcularamse os indicadores valor presente líquido (VPL) a taxas de desconto de 6 e de $10 \%$ (a primeira, uma taxa real compatível à aplicação do capital em caderneta de poupança, e a segunda, uma taxa mais apropriada à comparação com outras atividades agropecuárias alternativas) e taxa interna de retorno (TIR). A escolha de tais indicadores justificou-se pela vantagem de se considerar o efeito do tempo na correção do capital.

Calculados os indicadores de rentabilidade (VPL e TIR), realizou-se análise de sensibilidade (Melo et al., 1999), estabelecendose funções entre os preços de cada item do projeto e seus indicadores de rentabilidade calculados (Planilha Microsoft Excel). A análise consistiu em verificar o impacto que seria provocado por uma variação desfavorável de $10 \%$, em cada um dos itens de projeto, sobre seus indicadores de rentabilidade.
O passo seguinte consistiu em considerar o efeito do risco sobre os indicadores de rentabilidade do projeto, o que foi realizado através da técnica de simulação de Monte Carlo (Turban, 1993). Em virtude da dificuldade de se identificar a distribuição de probabilidades associada às variáveis do projeto, optou-se pela distribuição triangular, que é identificada a partir de apenas três de seus valores: um máximo, um mínimo e um mais provável (Lopes, 1992).

\section{RESULTADOS E DISCUSSÃO}

Os resultados financeiros (Tab. 2) foram concebidos prevendo-se a implantação total do sistema e não a simples ampliação de uma estrutura já existente. Os valores obtidos permitem concluir que o sistema proposto seria viável financeiramente.

Tabela 2. Indicadores financeiros de viabilidade para o sistema de produção de leite proposto

\begin{tabular}{lc} 
Indicador & Valor \\
\hline VPL a 6\% de desconto (R\$) & $197.880,00$ \\
VPL a 10\% de desconto (R\$) & $8.067,00$ \\
TIR (\%) & 10,22 \\
\hline VPL - valor presente líquido; TIR - taxa interna de retorno.
\end{tabular}

Os resultados do VPL indicam que os valores atuais para o projeto proposto, calculados com base nos custos de oportunidade do capital (Gomes, 1989), superariam os valores dos investimentos alternativos, com remunerações 
de 6 e 10\%, em R\$197.880,00 e 8.067,00, respectivamente.

A TIR, que representa a taxa de retorno sobre o capital empatado no projeto, durante a vida útil, mostra-se também expressiva. O valor desse indicador $(10,2 \%)$ é superior, por exemplo, à taxa que seria obtida aplicando-se o capital em caderneta de poupança, cujo rendimento real seria em torno de $6 \%$ ao ano.

Por meio da análise de sensibilidade (Tab. 3), constatou-se que os itens com maior impacto sobre a rentabilidade do projeto seriam, em ordem decrescente de importância: a) preço do leite, que é o principal item gerador de receita no sistema, evidenciando que pequenas alterações nos preços desse produto determinariam o sucesso ou fracasso com a atividade; b) compra e venda de animais, ressaltando a importância desse item nas exigências em sanidade, no seu potencial de produção (de acordo com o padrão racial dos animais), bem como na eficiência quanto à escolha dos animais e época adequada para sua compra e venda; c) mão-de-obra, salientando a necessidade de maior eficiência nesse item através da relação quantidade de mão-de-obra e a quantidade de leite produzida, sendo necessário planejar criteriosamente o sistema, levando em conta: distâncias entre as instalações, uso de áreas mecanizáveis destinadas à produção de volumosos, intensificação no uso das áreas disponíveis, organização quanto ao fluxo de serviços, bem como o pagamento por qualidade de serviço, dentre outros; d) alimentação concentrada para os animais adultos, reforçando o princípio de que, mesmo em sistemas em que o volumoso (pasto) participe com a maior parte na nutrição dos animais de produção, o item concentrado deve ser fornecido de acordo com o potencial de produção dos animais (divisão em lotes, por produção). Além disso, merece destaque a eficiência quanto ao custo por quantidade de proteína bruta $(\mathrm{PB})$ e de nutrientes digestíveis totais (NDT), fornecidos pelo concentrado, salientando cada vez mais o uso de resíduos agroindustriais de menor custo e a tendência preferencial à mistura da ração na propriedade.

Tabela 3. Resultado da análise de sensibilidade: redução percentual dos indicadores financeiros (VPL a $10 \%$ e TIR), decorrente de variações pessimistas isoladas de $10 \%$ nos valores monetários de cada item do projeto proposto

\begin{tabular}{|c|c|c|c|}
\hline Itens do projeto & VPL a $10 \%$ & TIR & Ordenação \\
\hline Manejo sanitário & $-75,10 \%$ & $-1,60 \%$ & 16 \\
\hline Forragem (pasto) & $-160,64 \%$ & $-3,42 \%$ & 7 \\
\hline Cana de açúcar corrigida & $-158,41 \%$ & $-3,38 \%$ & 8 \\
\hline Concentrado (animais adultos) & $-192,95 \%$ & $-4,11 \%$ & 5 \\
\hline Outros itens (alimentação) & $-78,85 \%$ & $-1,68 \%$ & 14 \\
\hline Leite para bezerras & $-106,51 \%$ & $-2,27 \%$ & 12 \\
\hline Inseminação & $-54,86 \%$ & $-1,17 \%$ & 18 \\
\hline Mão-de-obra & $-201,93 \%$ & $-4,30 \%$ & 4 \\
\hline Transporte do leite & $-38,94 \%$ & $-0,38 \%$ & 20 \\
\hline Combustível e energia & $-61,41 \%$ & $-1,31 \%$ & 17 \\
\hline Benfeitorias & $-158,81 \%$ & $-3,38 \%$ & 9 \\
\hline Formação de pastagens & $-75,98 \%$ & $-1,62 \%$ & 15 \\
\hline Formação do canavial & $-31,31 \%$ & $-0,67 \%$ & 19 \\
\hline Máquinas e equipamentos & $-81,63 \%$ & $-1,74 \%$ & 13 \\
\hline Aquisição de vacas & $-256,54 \%$ & $-5,38 \%$ & 2 \\
\hline Aquisição de rufiões & $-4,70 \%$ & $-0,10 \%$ & 21 \\
\hline Equipamentos para manejo dos animais & $-1,23 \%$ & $-0,03 \%$ & 23 \\
\hline Custo da terra nua + ITR & $-140,18 \%$ & $-2,98 \%$ & 10 \\
\hline Venda do leite & $-1246,05 \%$ & $-26,27 \%$ & 1 \\
\hline Venda de fêmeas excedentes & $-190,94 \%$ & $-4,08 \%$ & 6 \\
\hline Venda de vacas de descarte & $-224,04 \%$ & $-4,77 \%$ & 3 \\
\hline Venda de bezerros machos & $-120,02 \%$ & $-2,56 \%$ & 11 \\
\hline Venda de rufiões para o abate & $-3,15 \%$ & $-0,07 \%$ & 22 \\
\hline
\end{tabular}

$\mathrm{VPL}=$ valor presente líquido; $\mathrm{TIR}=$ taxa interna de retorno. 
Os resultados da simulação de Monte Carlo para os indicadores financeiros VPL a $6 \%$ e TIR encontram-se sintetizados nas Fig. 1 e 2, respectivamente. $\mathrm{Na}$ simulação realizada para $\mathrm{o}$ VPL, considerou-se a taxa de desconto de $6 \%$, de modo que os valores negativos para este indicador seriam interpretados como uma situação em que o sistema apresentaria rendimento inferior ao retorno proporcionado pela caderneta de poupança. Assim, conforme a Fig. 1, haveria 34 e $44 \%$ de probabilidade de o VPL atingir somas de $\mathrm{R} \$ 50.137,00$ e $\mathrm{R} \$ 57.691,00$, respectivamente, o que, por interpolação, equivaleria à probabilidade de $39 \%$ desse indicador ser nulo, ou seja, não haver vantagem adicional em aplicar o capital no projeto proposto.

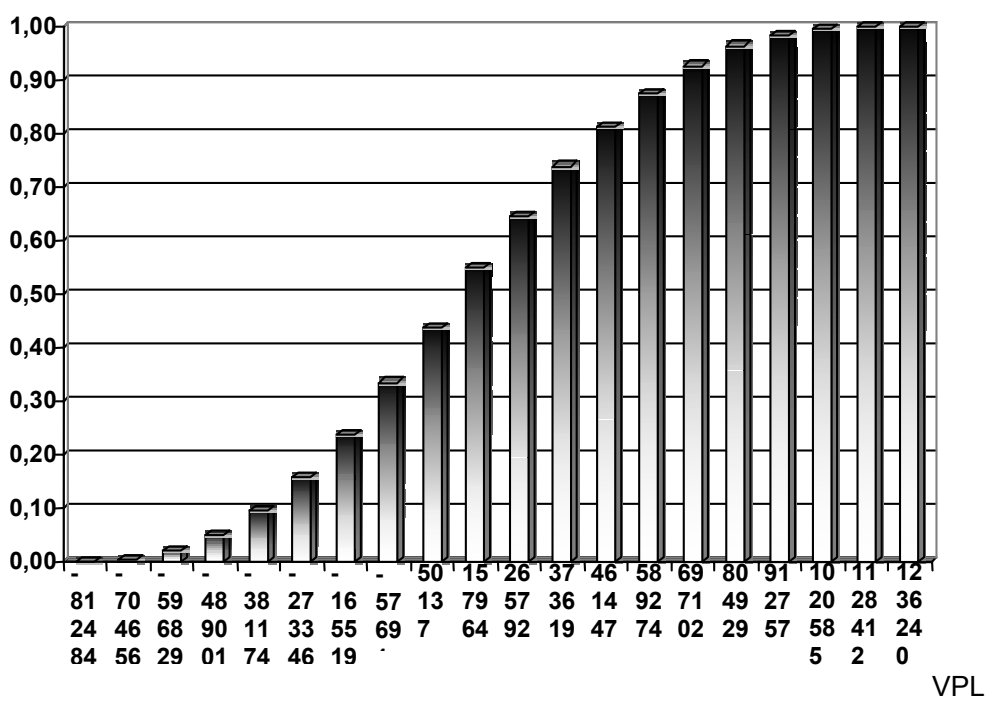

Figura 1. Distribuição acumulada das probabilidades para o valor presente líquido (VPL) do sistema de produção proposto, a taxa de desconto de $6 \%$.

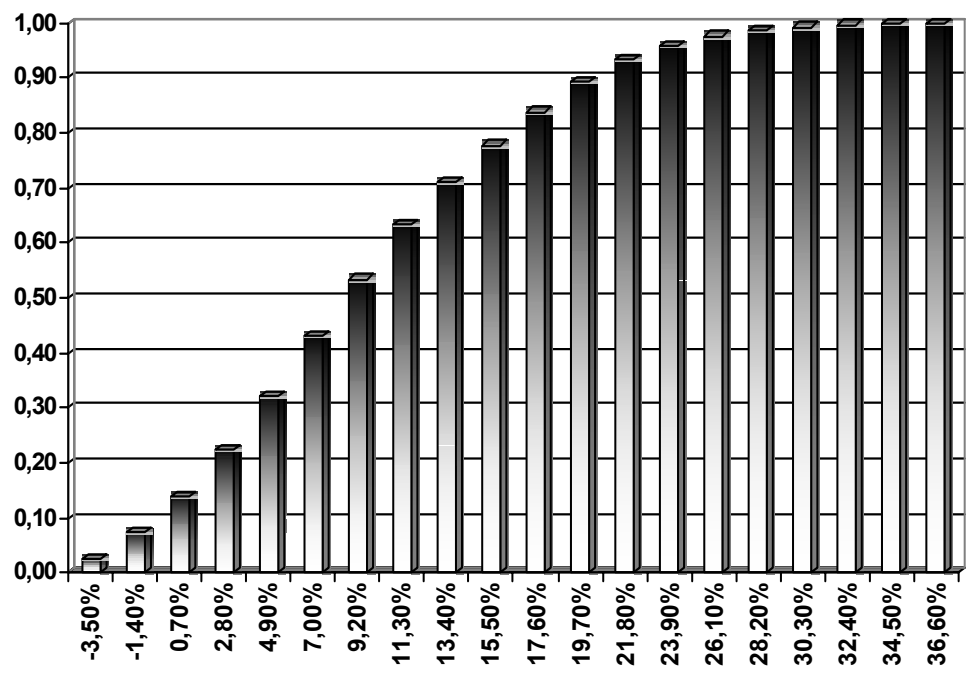

TIR

Figura 2. Distribuição acumulada de probabilidade para a taxa interna de retorno (TIR) do sistema de produção proposto. 
Quanto à TIR (Fig. 2), demonstrou-se que ela poderia assumir os valores de 4,9 e 7,0\%, com probabilidades de ocorrência, respectivamente, de 32 e $42 \%$. Interpolando-se estes valores, chegar-se-ia à conclusão de que a probabilidade da taxa interna de retorno menor que $6 \%$ ao ano seria de aproximadamente $38 \%$.

Essas probabilidades apresentaram-se bastante elevadas, evidenciando que, apesar de o projeto ser viável, haveria grande risco de o sistema não apresentar rentabilidade compatível sequer à da caderneta de poupança. $O$ fato torna-se preocupante, uma vez que o grande peso dos investimentos em ativos fixos, de elevada especificidade na atividade leiteira, impediriam que o pecuarista, diante de situações de baixa rentabilidade, pudesse sair da atividade, pois, se o fizesse, teria prejuízos ainda maiores.

\section{CONCLUSÕES}

Embora o sistema seja viável, há risco considerável de que a rentabilidade do projeto seja nula ou inferior ao rendimento da poupança. Portanto, o investimento no sistema é muito incerto. Além disso, destaca-se o fato de que, realizado o investimento, que na pecuária leiteira envolveria grande volume de ativos fixos, a saída da atividade acarretaria grandes perdas.

\section{REFERÊNCIAS BIBLIOGRÁFICAS}

ANUÁRIO DA PECUÁRIA BRASILEIRA. São Paulo, 2001. p.195-236

AZEVEDO FILHO, A.J.B.V. Análise econômica de projetos: "software" para situações determinísticas e de risco envolvendo simulação. 1988. 127f. Tese (Mestrado). Escola Superior de Agricultura de Queiroz, Universidade de São Paulo, Piracicaba, SP.
GOMES, S. T. O custo de produção de leite. Brasília: SNAB/MA, 1989. 66p.

LOPES, J.E.P. Análise econômica de contratos de integração usados no complexo agroindustrial avícola brasileiro. 1992. 94f. Tese (Mestrado). Universidade Federal de Viçosa, Viçosa, MG.

MELO, A.C.G.; REIS, M.S.; GORESTIN, B.G. Análise financeira de projetos de investimento sob enfoque de incertezas. In: SEMINÁRIO NACIONAL DE PRODUÇÃO E TRANSMISSÃO DE ENERGIA ELÉTRICA, 1999, Foz do Iguaçu. Anais... Foz do Iguaçu, 1999. p.1-6.

NORONHA, J.F. Projetos agropecuários: administração financeira, orçamento e viabilidade econômica. São Paulo: Atlas, 1987. 95p.

POTTER, L.; LOBATO, J.F.P.; MIELITZ NETO, C.G. Análises econômicas de sistemas de produção de novilhas de corte primíparas aos dois, três e quatro anos de idade. Rev. Bras. Zootec., v.29, p.861-870, 2000.

PREÇOS agrícolas, [s.1.]: IPEA, 2001. Disponível em: <www.ipeadata.gov.br>. Acessado em: 30/9/2001.

SCHREINER, D.F. Agricultural project investment analysis. In: TWEETEN, L. G. (Ed.). Agricultural policy analysis tools for economic development. Bolder, CO: Westview, 1989. p.238-277.

SILVA, S.C.; PASSANEZI, M. M. Planejamento do sistema de produção a pasto. In: PLANEJAMENTO da exploração leiteira. Piracicaba: FEALQ-USP, 1998. p.121-142.

TURBAN, E. Decision support and expert systems: management support systems. 3.ed. New York: Macmillan, 1993. 429p. 